\title{
ON BASE SIZES FOR ACTIONS OF FINITE CLASSICAL GROUPS
}

\author{
TIMOTHY C. BURNESS
}

\begin{abstract}
Let $G$ be a finite almost simple classical group and let $\Omega$ be a faithful primitive non-standard $G$-set. A base for $G$ is a subset $B \subseteq \Omega$ whose pointwise stabilizer is trivial; we write $b(G)$ for the minimal size of a base for $G$. A well-known conjecture of Cameron and Kantor asserts that there exists an absolute constant $c$ such that $b(G) \leqslant c$ for all such groups $G$, and the existence of such an undetermined constant has been established by Liebeck and Shalev. In this paper we prove that either $b(G) \leqslant 4$, or $G=\mathrm{U}_{6}(2) \cdot 2, G_{\omega}=\mathrm{U}_{4}(3) \cdot 2^{2}$ and $b(G)=5$. The proof is probabilistic, using bounds on fixed point ratios.
\end{abstract}

\section{Introduction}

A base for a finite permutation group $G$ on a set $\Omega$ is a subset $B \subseteq \Omega$ whose pointwise stabilizer is trivial. Bases are of interest in several different fields. For example, they play an important rôle in computational group theory because each element of $G$ is uniquely determined by its action on $B$ and can therefore be stored as a $|B|$-tuple rather than a $|\Omega|$-tuple. In this respect, small bases are of particular interest. We write $b(G)$ for the minimal size of a base for $G$. Determining $b(G)$ is a fundamental problem in permutation group theory.

In this paper we obtain upper bounds on base sizes for primitive actions of almost simple classical groups. A main motivation comes from a conjecture of Cameron and Kantor [8, 9] which was settled in the affirmative by Liebeck and Shalev [22]. The conjecture concerns non-standard actions of finite almost simple groups.

Definition 1. Let $G$ be a finite almost simple group with socle $G_{0}$. Then a transitive action of $G$ on a set $\Omega$ is said to be standard if one of the following holds:

(i) $G_{0}=A_{n}$ and $\Omega$ is an orbit of subsets or partitions of $\{1, \ldots, n\}$;

(ii) $G$ is a classical group in a subspace action.

Naturally, an action of $G$ which is equivalent to a standard action of an isomorphic group is also said to be a standard action of $G$. Non-standard actions are defined accordingly. Roughly

2000 Mathematics Subject Classification 20B15 (primary), 20P05 (secondary). 
speaking, if $G$ is a classical group with natural module $V$ then a transitive action of $G$ is a subspace action if $G$ permutes subspaces of $V$, or pairs of subspaces of complementary dimension (see Definition 2).

The Cameron-Kantor Conjecture asserts that there exists an absolute constant $c$ such that $b(G) \leqslant c$ for any almost simple primitive permutation group $G$ in a faithful non-standard action. In general, the orders of the groups in (i) and (ii) are not bounded from above by a fixed polynomial function of their degree and thus $b(G)$ is unbounded for standard actions.

The case $G_{0}=A_{n}$ was settled by Cameron and Kantor $[\mathbf{9}]$. By an elementary counting argument they showed that almost every pair of points in a non-standard $G$-set form a base for $G$ as $|G|$ tends to infinity. In recent work [14], Guralnick and Saxl have proved that $b(G)=2$ if $n>12$, and it quickly follows that $b(G) \leqslant 3$ for all $n$. Combined with work of James [16], the primitive actions of alternating and symmetric groups which admit a base of size two have been completely determined.

For classical groups, the existence of an absolute constant $c$ which satisfies the conclusion of the Cameron-Kantor Conjecture is established in $[\mathbf{2 2}, 1.3]$ but the proof does not yield an explicit value. However, some recent progress has been made in this direction. In [23, 1.11] Liebeck and Shalev show that if $G$ is a classical group in a non-subspace action then $b(G) \leqslant 3$ as $|G|$ tends to infinity. Although this asymptotic result relies on the additional hypothesis that the dimension of the natural module is greater than fifteen, it is essentially best possible because there exist non-standard primitive actions of classical groups of arbitrarily large rank with $b(G)>2$. In addition, some non-asymptotic results have been obtained in specific cases. For example, the action of $\operatorname{PGL}_{2 n}(q)$ on the cosets of $\operatorname{PGSp}_{2 n}(q)$ is studied in [12] where it is shown that $b(G)=3$ if $n>3$. Precise base size results for partition actions of linear groups can be found in $[\mathbf{1 7}]$.

The main feature of Theorem 1 below is an explicit bound on $b(G)$ which is valid for all non-standard actions of finite almost simple classical groups. Here $H=G_{\omega}$ denotes the point stabilizer in $G$ of an arbitrary element $\omega$ from the permutation domain.

THEOREM 1. Let $G$ be a finite almost simple classical group in a faithful primitive nonstandard action. Then either $b(G) \leqslant 4$, or $G=\mathrm{U}_{6}(2) .2, H=\mathrm{U}_{4}(3) .2^{2}$ and $b(G)=5$.

REMARK 1. In the statement of Theorem 1, we omit any action of $G$ which is equivalent to a standard action of an isomorphic group. A list of these standard actions is presented in Table 1. 
In the forthcoming paper [7] we consider base sizes for primitive actions of exceptional groups. A concise version of our main result states that if $G$ is a finite almost simple primitive exceptional group of Lie type then $b(G) \leqslant 6$ (see [7] for a more detailed statement). In view of Theorem 1, we get

Corollary 1. If $G$ is a finite almost simple group of Lie type in a faithful primitive non-standard action then $b(G) \leqslant 6$.

The proof of the Cameron-Kantor Conjecture for classical groups relies on an existence result $[\mathbf{2 2}$, Theorem $(\star)]$. This theorem states that there exists an absolute constant $\epsilon>0$ so that if $G$ is any finite almost simple classical group in a primitive non-subspace action then

$$
\operatorname{fpr}(x, \Omega)<\left|x^{G}\right|^{-\epsilon}
$$

for all elements $x \in G$ of prime order, where $\operatorname{fpr}(x, \Omega)$ denotes the fixed point ratio of $x$, i.e. the proportion of points in the permutation domain $\Omega$ which are fixed by $x$. The connection to base sizes arises in the following way. If $Q(G, c)$ denotes the probability that a randomly chosen $c$-tuple in $\Omega$ does not form a base for $G$ then

$$
Q(G, c) \leqslant \sum_{i=1}^{k}\left|x_{i}^{G}\right| \cdot \operatorname{fpr}\left(x_{i}, \Omega\right)^{c},
$$

where $x_{1}, \ldots, x_{k}$ represent the distinct $G$-classes of elements of prime order in $G$. Of course, $G$ admits a base of size $c$ if and only if $Q(G, c)<1$. In the proof of $[\mathbf{2 2}, 1.3]$ it is shown that $b(G) \leqslant 11 \epsilon^{-1}$, an undetermined bound since $[\mathbf{2 2}$, Theorem $(\star)]$ is strictly an existence result.

The main theorem of $[\mathbf{3}]$ states that (1.1) holds with $\epsilon=1 / 2-1 / n-\iota$, where $n$ is a natural number associated to each almost simple classical group and $\iota \geqslant 0$ is a known constant depending on $G$ and $\Omega$ with the property that $\iota \rightarrow 0$ as $n \rightarrow \infty$ (the precise values of $\iota$ are listed in [3, Table 1]). In general, $n$ is simply the dimension of the natural $G$-module - see Remark 2.

In applying (1.1) and (1.2), the zeta-type function

$$
\eta_{G}(t)=\sum_{C \in \mathscr{C}}|C|^{-t}
$$

arises naturally in the proof of $[\mathbf{2 2}, 1.3]$, where $t \in \mathbb{R}$ and $\mathscr{C}$ is the set of conjugacy classes in $G$ of elements of prime order. Evidently there exists a real number $T_{G} \in(0,1)$ such that $\eta_{G}\left(T_{G}\right)=1$ and applying [3, Theorem 1] we deduce that $G$ admits a base of size $c$ if

$$
T_{G}<c(1 / 2-1 / n-\iota)-1 .
$$


In this way, we can bound $b(G)$ by bounding the function $T_{G}$. Indeed, Proposition 2.3 states that $T_{G}<1 / 3$ if $n \geqslant 6$ and Theorem 1 quickly follows, with the exception of a short list of cases. For these remaining cases we either establish a stronger upper bound for $T_{G}$ and obtain the desired result via (1.3), or we derive an explicit upper bound $Q(G, c) \leqslant F(q)$ via (1.2) with the property that $F(q)<1$ for all possible values of $q$. Here the computer package GAP [11] is a useful tool when dealing with groups of small rank over small fields. In the proof of Theorem 1 we provide detailed results for the cases $G_{0}=\operatorname{PSp}_{4}(q)^{\prime}$ and $\operatorname{PSL}_{n}^{\epsilon}(q)$ with $n \leqslant 5$ (see Proposition 4.1 and Tables 5 and 6).

LAYOUT. This paper is organized as follows. In the next section we record some preliminary results which we require for the proof of Theorem 1. In particular, we show that $G$ admits a base of size $c$ if (1.3) holds (Proposition 2.2) and we prove that $T_{G}<1 / 3$ if $n \geqslant 6$ (Proposition 2.3). In Section 3 we establish Theorem 1 for $n \geqslant 6$; the remaining small rank cases are considered in Section 4.

Notation. Our notation and terminology for classical groups is standard (see [20] and [3], for example). In particular, we write $\operatorname{PSL}_{n}^{+}(q)=\operatorname{PSL}_{n}(q)=\mathrm{L}_{n}(q)$ and $\operatorname{PSL}_{n}^{-}(q)=\operatorname{PSU}_{n}(q)=$ $\mathrm{U}_{n}(q)$. We adopt the terminology of $[\mathbf{1 3}, 2.5 .13]$ for the various automorphisms of simple groups of Lie type. In addition, $G^{\prime}$ denotes the derived subgroup of a group $G$; $G^{m}$ is the direct product of $m$ copies of $G$; H.G denotes an (arbitrary) extension of a group $H$ by $G$, while $i_{r}(G)$ is the number of elements of order $r$ in $G$. We write $n$ or $C_{n}$ for a cyclic group of order $n$, while $p^{m}$ denotes an elementary abelian $p$-group of order $p^{m}$ for a prime $p$ and $D_{n}$ is the dihedral group of order $n$. If $\mathbb{F}$ is a field then $\overline{\mathbb{F}}$ will denote the algebraic closure of $\mathbb{F}$. Also, we write $(a, b)$ for the highest common factor of the integers $a$ and $b$, while $\delta_{i, j}$ is the usual Kronecker delta. We use $\log n$ to denote $\log _{e} n$ and we write $\mathbb{F}_{q}=\mathrm{GF}(q)$ for the field of $q$ elements.

\section{Preliminary results}

Let $G$ be a finite almost simple classical group over $\mathbb{F}_{q}$, where $q=p^{f}$ and $p$ is prime, with socle $G_{0}$ and natural module $V$. In studying actions of classical groups, it is natural to distinguish between those actions which permute subspaces of the natural module and those which do not. In this paper we are interested in bases for non-subspace actions. This notion was introduced by Liebeck and Shalev in [22].

Definition 2. A subgroup $H$ of $G$ not containing $G_{0}$ is a subspace subgroup if for each maximal subgroup $M$ of $G_{0}$ containing $H \cap G_{0}$ one of the following holds: 
(i) $M$ is the stabilizer in $G_{0}$ of a proper non-zero subspace $U$ of $V$, where $U$ is totally singular, non-degenerate, or, if $G_{0}$ is orthogonal and $p=2$, a non-singular 1-space ( $U$ can be any subspace if $\left.G_{0}=\operatorname{PSL}(V)\right)$;

(ii) $M=\mathrm{O}_{2 m}^{ \pm}(q)$ if $\left(G_{0}, p\right)=\left(\operatorname{Sp}_{2 m}(q)^{\prime}, 2\right)$.

A faithful transitive action of $G$ on a set $\Omega$ is a subspace action if the point stabilizer $G_{\omega}$ of an element $\omega \in \Omega$ is a subspace subgroup of $G$. Non-subspace subgroups and actions are defined accordingly.

Recall the definition of a standard action from the Introduction (see Definition 1). Evidently, every subspace action of $G$ is standard, but the converse does not hold because a non-subspace action of $G$ may be equivalent to a standard action of an isomorphic group. The primitive standard actions that arise in this way are listed in Table 1 . Here $H$ is the stabilizer in $G$ of an element in the permutation domain; following [20], the type of $H$ provides an approximate group-theoretic structure for $H \cap \operatorname{PGL}(V)$, where $V$ is the natural $G_{0}$-module. An $l$-subspace is an $l$-dimensional subspace of the natural module for the relevant classical group isomorphic to $G_{0}$; an $\epsilon 4$-subspace for $\Omega_{5}(q)$ is a non-degenerate 4 -subspace whose stabilizer in $\Omega_{5}(q)$ is an orthogonal group of type $\mathrm{O}_{4}^{\epsilon}(q)$.

\begin{tabular}{llll}
\hline$G_{0}$ & type of $H$ & conditions & equivalent action \\
\hline $\mathrm{P} \Omega_{8}^{+}(q)$ & $\Omega_{7}(q)$ & $H$ irreducible & $\mathrm{P} \Omega_{8}^{+}(q)$ on non-singular 1-subspaces \\
$\mathrm{P} \Omega_{8}^{+}(q)$ & $\mathrm{Sp}_{4}(q) \otimes \mathrm{Sp}_{2}(q)$ & & $\mathrm{P} \Omega_{8}^{+}(q)$ on non-singular 3 -subspaces \\
$\mathrm{PSL}_{4}^{\epsilon}(q)$ & $\mathrm{Sp}_{4}(q)$ & & $\mathrm{P} \Omega_{6}^{\epsilon}(q)$ on non-singular 1-subspaces \\
$\mathrm{L}_{4}(q)$ & $\mathrm{GL}_{2}\left(q^{2}\right)$ & $q=2$ & $A_{8}$ on -element subsets of $\{1, \ldots, 8\}$ \\
$\mathrm{L}_{4}(q)$ & $A_{7}$ & $q=2$ & $A_{8}$ on 1-element subsets of $\{1, \ldots, 8\}$ \\
$\mathrm{PSp}_{4}(q)^{\prime}$ & $\mathrm{Sp}_{2}(q) 2 S_{2}$ & & $\Omega_{5}(q)$ on +4 -subspaces \\
& $\mathrm{Sp}_{2}\left(q^{2}\right)$ & & $\Omega_{5}(q)$ on -4 -subspaces \\
& $2^{4} \cdot \mathrm{O}_{4}^{-}(2)$ & $q=3$ & $\mathrm{U}_{4}(2)$ on totally singular 2 -subspaces \\
& $\mathrm{GL}_{2}\left(q_{0}\right)$ & $q=q_{0}^{2}$ & $\Omega_{4}^{-}\left(q_{0}\right)$ on non-singular 1-subspaces \\
& $2^{2} \cdot \mathrm{O}_{2}^{-}(2)$ & $q=7$ & $\mathrm{~L}_{3}(2)$ on 1-subspaces \\
& & $q=5$ & $A_{5}$ on 1-element subsets of $\{1, \ldots, 5\}$ \\
& $A_{5}(q)$ & $q=9$ & $A_{6}$ on 1-element subsets of $\{1, \ldots, 6\}$ \\
\hline
\end{tabular}

TABle 1. Some standard actions

The main theorem on the subgroup structure of finite classical groups is due to Aschbacher. In [1] , eight collections of subgroups of $G$ are defined, labelled $\mathscr{C}_{i}$ for $1 \leqslant i \leqslant 8$, and in general it is shown that if $H$ is a maximal subgroup of $G$ not containing $G_{0}$ then either $H$ is contained in one of these $\mathscr{C}_{i}$ collections or it belongs to a family of almost simple groups which act 
irreducibly on the natural $G_{0}$-module. We refer the reader to $[\mathbf{2 0}]$ for detailed information on these subgroup collections. Due to the existence of certain outer automorphisms, a small additional collection arises when $G_{0}=\operatorname{Sp}_{4}(q)^{\prime}\left(q\right.$ even) or $\mathrm{P}_{8}^{+}(q)$ (see $[\mathbf{1}, \S 14]$ and $\left.[\mathbf{1 8}, \S 4]\right)$. Roughly speaking, a maximal subgroup is non-subspace unless it is a member of the collection $\mathscr{C}_{1}$ or is a particular example of a subgroup in $\mathscr{C}_{8}$, where we follow [20] in labelling the various $\mathscr{C}_{i}$ collections.

In $[\mathbf{3}, \mathbf{4}, \mathbf{5}, \mathbf{6}]$ we studied fixed point ratios for non-subspace actions of finite classical groups. Let $\Omega$ be a $G$-set and recall that the fixed point ratio of $x \in G$, which we denote by $\operatorname{fpr}(x, \Omega)$, is the proportion of points in $\Omega$ which are fixed by $x$. If $G$ acts transitively on $\Omega$ then it is easy to see that

$$
\operatorname{fpr}(x, \Omega)=\frac{\left|x^{G} \cap H\right|}{\left|x^{G}\right|},
$$

where $H=G_{\omega}$ for some $\omega \in \Omega$. In [22], Liebeck and Shalev prove that there exists an absolute constant $\epsilon>0$ so that if $G$ is any finite almost simple classical group in a primitive nonsubspace action then $\operatorname{fpr}(x, \Omega)<\left|x^{G}\right|^{-\epsilon}$ for all elements $x \in G$ of prime order. This result plays a major rôle in their proof of the Cameron-Kantor Conjecture (see Section 1) and it finds a wide range of other interesting applications in $[\mathbf{2 2}]$. The main theorem of $[\mathbf{3}]$ states that the Liebeck-Shalev result holds with an explicit constant $\epsilon \approx 1 / 2$.

TheOREM 2.1 ([3, Theorem 1]). Let $G$ be a finite almost simple classical group over $\mathbb{F}_{q}$ and let $\Omega$ be a faithful primitive non-subspace $G$-set. Then

$$
\operatorname{fpr}(x, \Omega)<\left|x^{G}\right|^{-\frac{1}{2}+\frac{1}{n}+\iota}
$$

for all $x \in G$ of prime order, where $\iota=\iota(G, \Omega) \geqslant 0$ is a known constant.

REMARK 2. In most cases $\iota=0$; the precise values are listed in [3, Table 1]. The integer $n$ in the statement of Theorem 2.1 is defined as follows: if $G_{0}=\operatorname{Sp}_{4}(2)^{\prime}$ or $\mathrm{L}_{3}(2)$ then $n=2$, otherwise $n$ is the minimal dimension of a non-trivial irreducible $K \widehat{G}_{0}$-module, where $\widehat{G}_{0}$ is a covering group of $G_{0}$ and $K=\overline{\mathbb{F}}_{q}$, i.e. $n$ is the dimension of the natural module for $G_{0}$, whenever this is well-defined.

Next we associate a "zeta function" to $G$ which encodes the sizes of the conjugacy classes of elements of prime order. 
Definition 3. Let $\mathscr{C}$ be the set of conjugacy classes of elements of prime order in $G$. For $t \in \mathbb{R}$ define

$$
\eta_{G}(t)=\sum_{C \in \mathscr{C}}|C|^{-t}
$$

and fix $T_{G} \in(0,1)$ such that $\eta_{G}\left(T_{G}\right)=1$.

REMARK 3. We refer the reader to $[\mathbf{2 3}, 1.10]$ for results on the asymptotic behaviour of $\eta_{G}(t)$. Note that in summing over all conjugacy classes, the definition of $\eta_{G}(t)$ in $[\mathbf{2 3}]$ differs slightly from ours.

Proposition 2.2. If $\Omega$ is a faithful primitive non-subspace $G$-set and $T_{G}<c(1 / 2-1 / n-$ ८) 1 then $b(G) \leqslant c$.

Proof. We follow the proof of $[\mathbf{2 3}, 1.11]$. Let $x_{1}, \ldots, x_{k}$ be representatives for the $G$-classes of elements of prime order in $G$ and let $Q(G, c)$ be the probability that a randomly chosen $c$-tuple of points in $\Omega$ is not a base for $G$. Evidently, $G$ admits a base of size $c$ if and only if $Q(G, c)<1$. Now, a $c$-tuple in $\Omega$ fails to be a base if and only if it is fixed by an element $x \in G$ of prime order. The probability that a random $c$-tuple is fixed by $x$ is precisely $\operatorname{fpr}(x, \Omega)^{c}$, and since fixed point ratios are constant on conjugacy classes (see (2.1)) we deduce that

$$
Q(G, c) \leqslant \sum_{x \in \mathscr{P}} \operatorname{fpr}(x, \Omega)^{c}=\sum_{i=1}^{k}\left|x_{i}^{G}\right| \cdot \operatorname{fpr}\left(x_{i}, \Omega\right)^{c},
$$

where $\mathscr{P}$ is the set of elements of prime order in $G$. Hence Theorem 2.1 gives

$$
Q(G, c) \leqslant \sum_{i=1}^{k}\left|x_{i}^{G}\right|^{c(-1 / 2+1 / n+\iota)+1}=\eta_{G}(c(1 / 2-1 / n-\iota)-1)
$$

and the desired result follows since $\eta_{G}(t)<1$ for all $t>T_{G}$.

Definition 4. For $x \in \operatorname{PGL}(V)$ let $\hat{x}$ be a pre-image of $x$ in $\operatorname{GL}(V)$ and define

$$
\nu(x):=\min \left\{\operatorname{dim}[\bar{V}, \lambda \hat{x}]: \lambda \in K^{*}\right\}
$$

where $\bar{V}=V \otimes K$ and $K=\overline{\mathbb{F}}_{q}$. Note that $\nu(x)$ is equal to the codimension of the largest eigenspace of $\hat{x}$ on $\bar{V}$ and thus $\nu(x)>0$ if $x \neq 1$.

The next result allows us to effectively apply Proposition 2.2 .

Proposition 2.3. If $n \geqslant 6$ then $T_{G}<1 / 3$. 
Proof. Let $\bar{G}$ be a simple classical algebraic group over $\overline{\mathbb{F}}_{q}$ of adjoint type which admits a Frobenius morphism $\sigma$ such that the finite subgroup $\bar{G}_{\sigma}:=\left\{g \in \bar{G}: g^{\sigma}=g\right\}$ has socle $G_{0}$. In the terminology of $[\mathbf{1 3}], \bar{G}_{\sigma}$ is the group of inner-diagonal automorphisms of $G_{0}$. For now, let us assume $G_{0}=\operatorname{PSp}_{n}(q)$. For $1 \leqslant s \leqslant n-1$, let $k_{s, u}$ (respectively, $k_{s, s}$ ) denote the number of distinct $G$-classes of unipotent (respectively semisimple) elements $x \in G$ of prime order with $\nu(x)=s$, and write $c(s)$ for the size of the smallest $G$-class of elements $x \in G$ of prime order with $\nu(x)=s$. In addition, let $\pi$ be the set of distinct prime divisors of $\log _{p} q$ and for $r \in \pi$ let $\mathscr{F}_{r}$ be the set of $G$-classes of field automorphisms of order $r$. Then

$$
\eta_{G}(t) \leqslant \sum_{r \in \pi} \sum_{C \in \mathscr{F}_{r}}|C|^{-t}+\sum_{s=1}^{n-1}\left(k_{s, u}+k_{s, s}\right) \cdot c(s)^{-t} .
$$

Set $d=(2, q-1)$. We claim that the following bounds hold:

(i) $|\pi| \leqslant \log \left(\log _{p} q+2\right)$ and $\left|\mathscr{F}_{r}\right| \leqslant d\left(\log _{p} q-1\right)$ for all $r \in \pi$;

(ii) $|C|>\frac{1}{2 d} q^{\frac{1}{4} n(n+1)}$ for all $C \in \mathscr{F}_{r}$;

(iii) $k_{s, u}<d .2^{s+\sqrt{s}}$

(iv) $k_{s, s}<q^{\frac{1}{2}(s+1)} \cdot \frac{1}{4} n(n+2) \log q$;

(v) $c(s)>\frac{1}{4 d}(q+1)^{-1} q^{\max \left(s(n-s), \frac{1}{2} n s\right)+1}=d(s)$.

First consider (i). Any $N \in \mathbb{N}$ has fewer than $\log (N+2)$ distinct prime divisors, so $|\pi| \leqslant$ $\log \left(\log _{p} q+2\right)$. Further, if $r \in \pi$ then $\left|\mathscr{F}_{r}\right| \leqslant d(r-1)$ (see $\left.[\mathbf{1 3}, 4.9 .1]\right)$ and hence (i) holds since $r$ divides $\log _{p} q$. Part (ii) follows immediately from $[4,3.48]$ and we observe that (i) and (ii) imply that

$$
\sum_{r \in \pi} \sum_{C \in \mathscr{F}_{r}}|C|^{-t}<d\left(\log _{p} q-1\right) \cdot \log \left(\log _{p} q+2\right) \cdot\left(\frac{1}{2 d} q^{\frac{1}{4} n(n+1)}\right)^{-t}=\Gamma_{1}(n, q, t) .
$$

According to $[\mathbf{4},(9)]$, there are fewer than $2^{s+\sqrt{s}}$ distinct $\bar{G}_{\sigma}$-classes of unipotent elements $x$ with $\nu(x)=s$ and thus (iii) holds since each $\bar{G}_{\sigma}$-class can split into at most $d=\left|\bar{G}_{\sigma}: G_{0}\right|$ distinct $G$-classes. Next consider (iv). Fix a prime $r \neq p$ which divides $\left|\bar{G}_{\sigma}\right|$. By $[\mathbf{4}, 3.40]$, there are at most $q^{(s+1) / 2}$ distinct $G$-classes of elements $x \in G$ of order $r$ with $\nu(x)=s$ and thus it suffices to show that there are fewer than $\frac{1}{4} n(n+2) \log q$ possibilities for $r$. Since $r \neq p$, it follows that $r$ divides $\prod_{i=1}^{n / 2}\left(q^{2 i}-1\right)<q^{n(n+2) / 4}$ and thus (iv) holds since any integer $N>6$ has fewer than $\log N$ distinct prime divisors. Finally, the bound in (v) follows at once from $[\mathbf{4}$, $3.38]$ and we conclude that $\sum_{s}\left(k_{s, u}+k_{s, s}\right) . c(s)^{-t}<\Gamma_{2}(n, q, t)$, where

$$
\Gamma_{2}(n, q, t)=\sum_{s=1}^{n-1}\left[\left(d .2^{s+\sqrt{s}}+q^{\frac{1}{2}(s+1)} \cdot \frac{1}{4} n(n+2) \log q\right) \cdot\left(\frac{1}{4 d}(q+1)^{-1} q^{\alpha}\right)^{-t}\right]
$$


and $\alpha=\max \left(s(n-s), \frac{1}{2} n s\right)+1$, hence $\eta_{G}(t)<\Gamma_{1}(n, q, t)+\Gamma_{2}(n, q, t)$. For $n \geqslant 8$, one can check that $\Gamma_{1}(n, q, 1 / 3)+\Gamma_{2}(n, q, 1 / 3)<1$ with the exception of a short list of cases for which both $n$ and $q$ are small (there are no exceptions if $n>30$ or $q>43$ ). Here we can calculate $\rho(n, q)$ precisely, where $\rho(n, q)+1$ is the number of distinct prime divisors of $\left|\operatorname{Sp}_{n}(q)\right|$, and thus replace the term $\frac{1}{4} n(n+2) \log q$ by $\rho(n, q)$ in the above bound (iv) for $k_{s, s}$. Then the subsequent bound for $\eta_{G}(t)$ is sufficient with a much shorter list of exceptions; these cases can be checked by hand with the aid of $[\mathbf{4}, \S \S 3.2-3.5]$. For example, if $(n, q)=(8,2)$ then $G=\operatorname{Sp}_{8}(2)$ and $T_{G}<.228$ since the possibilities for $|C|$ are as follows, where $C$ is a conjugacy class in $G$ containing elements of prime order $r$.

\begin{tabular}{ll}
\hline$r$ & $|C|$ \\
\hline 2 & $255,5355,16065,64260,321300,963900$ \\
3 & $10880,609280,3655680,12185600$ \\
5 & 13160448,157925376 \\
7 & 1128038400 \\
17 & 2786918400 (2 classes) \\
\hline
\end{tabular}

If $G_{0}=\mathrm{PSp}_{6}(q)$ then the more accurate bound

$$
\begin{aligned}
\eta_{G}(t)< & 2\left(\frac{1}{2}\left(q^{6}-1\right)\right)^{-t}+\left(2+\frac{q}{2} \log \left(q^{2}-1\right)\right) \cdot\left(\frac{1}{4}(q+1)^{-1} q^{11}\right)^{-t} \\
& +\left(\left(q^{2}+1\right)\left(q^{6}-1\right)\right)^{-t}+\left(6+\frac{q}{2} \log \left(q^{2}-1\right)\right) \cdot\left(\frac{1}{4}(q+1)^{-1} q^{13}\right)^{-t} \\
& +q^{2} \log \left(q^{2}+1\right)\left(\frac{1}{8} q^{14}\right)^{-t}+\frac{1}{3} q^{3} \log \left(q^{6}-1\right) \cdot\left(\frac{1}{4} q^{18}\right)^{-t} \\
& +2\left(\log _{p} q-1\right) \cdot \log \left(\log _{p} q+2\right) \cdot\left(\frac{1}{4} q^{\frac{21}{2}}\right)^{-t}
\end{aligned}
$$

is sufficient unless $q=2$ or 3 . These two cases can be checked directly; we find that $T_{G}<.307$ when $q=2$, while $T_{G}<.240$ if $q=3$.

A very similar argument applies when $G_{0}=\operatorname{PSL}_{n}^{\epsilon}(q)$ or $\Omega_{n}^{\epsilon}(q)$; we leave the details to the reader (see $[4, \S 3.5]$ for relevant information on graph and graph-field automorphisms).

REMARK 4. For certain $G$ we require a slightly stronger upper bound on $T_{G}$. To be precise, we need $T_{G}<1 / 5$ if $G_{0}=\operatorname{PSL}_{10}^{\epsilon}(q)$, and $T_{G}<4 / 15$ if $G_{0}=\mathrm{P}_{n}^{\epsilon}(q)$ and $n \geqslant 12$ is even. These bounds can be established by arguing as in the proof of Proposition 2.3.

The next two basic lemmas will be useful in the proof of Theorem 1.

Lemma 2.4. Let $G$ be a transitive permutation group on a finite set $\Omega$ and write $H=G_{\omega}$ for some $\omega \in \Omega$. Suppose $x_{1}, \ldots, x_{m}$ represent distinct $G$-classes such that $\sum_{i}\left|x_{i}^{G} \cap H\right| \leqslant A$ 
and $\left|x_{i}^{G}\right| \geqslant B$ for all $1 \leqslant i \leqslant m$. Then

$$
\sum_{i=1}^{m}\left|x_{i}^{G}\right| \cdot \operatorname{fpr}\left(x_{i}, \Omega\right)^{c} \leqslant B(A / B)^{c}
$$

for all $c \in \mathbb{N}$.

Proof. Without loss we may assume $\left|x_{i}^{G}\right| \geqslant\left|x_{i+1}^{G}\right|$. For $1 \leqslant i \leqslant m-1$ set $a_{i}=\left|x_{i}^{G} \cap H\right|$ and $b_{i}=\left|x_{i}^{G}\right|-B$. Then in view of (2.1) we have

$$
\begin{aligned}
\sum_{i=1}^{m}\left|x_{i}^{G}\right| \cdot \operatorname{fpr}\left(x_{i}, \Omega\right)^{c} & \leqslant B\left(\frac{A-\sum_{i} a_{i}}{B}\right)^{c}+\sum_{i}\left(B+b_{i}\right)\left(\frac{a_{i}}{B+b_{i}}\right)^{c} \\
& \leqslant B^{1-c}\left(\left(A-\sum_{i} a_{i}\right)^{c}+\sum_{i} a_{i}^{c}\right)
\end{aligned}
$$

and the desired bound follows.

Lemma 2.5. Let $G$ be a permutation group on a finite set $\Omega$. Then $b(G) \geqslant(\log |G|) /(\log |\Omega|)$.

Proof. If $B \subseteq \Omega$ is a base then each element of $G$ is uniquely determined by its action on $B$, whence $|G| \leqslant|\Omega|^{|B|}$ and the result follows.

\section{Proof of Theorem 1, Part I: $n \geqslant 6$}

We begin the proof of Theorem 1 by assuming $n \geqslant 6$. We partition this section into three subsections, according to the possibilities for $G_{0}$.

3.1. $G_{0}=\operatorname{PSp}_{n}(q)$

Lemma 3.1. Suppose $G_{0}=\operatorname{Sp}_{6}(q)$ and $H$ is an irreducible subgroup of type $\mathrm{G}_{2}(q)$, where $q$ is even. Then $b(G)=4$.

Proof. Here $G=G_{0} \cdot\langle\phi\rangle$ and $H=G_{2}(q) \cdot\langle\phi\rangle$, where $\phi$ is a (possibly trivial) field automorphism of $G_{0}$. Let $\rho: \Omega_{8}^{+}(q) \rightarrow \Omega_{8}^{+}(q)$ be an irreducible spin representation and view $G_{0}$ as the stabilizer in $\Omega_{8}^{+}(q)$ of a 1-dimensional non-singular subspace of the natural module $\tilde{V}$ for $\Omega_{8}^{+}(q)$. Then the action of $G$ on $\Omega$ is equivalent to the action of $\rho\left(G_{0}\right) \cdot\langle\phi\rangle$ on the set of 1-dimensional non-singular subspaces of $\tilde{V}$. Therefore, it suffices to show that there exist four non-singular vectors $v_{1}, \ldots, v_{4}$ in $\tilde{V}$ such that $\bigcap_{i} G_{\left\langle v_{i}\right\rangle}=\{1\}$, while the intersection of any three such stabilizers is non-trivial. In the proof we adopt the notation of [2] for labelling representatives of unipotent classes of involutions in orthogonal groups.

Fix non-singular vectors $v_{1}, \ldots, v_{4}$ which span a -4 -subspace $W$ of $\tilde{V}$, so $W$ is a 4-dimensional non-degenerate subspace and the restriction of the corresponding quadratic form on $\tilde{V}$ to $W$ 
has defect -1 . By carefully choosing the $v_{i}$, we may assume that the subspaces $\left\langle v_{i}\right\rangle$ are not simultaneously fixed by a field automorphism of $G_{0}$. We claim that $\bigcap_{i} G_{\left\langle v_{i}\right\rangle}=\{1\}$. Seeking a contradiction, suppose $x \in \bigcap_{i} G_{\left\langle v_{i}\right\rangle}$ has prime order $r$. Then $x \in G_{0}$ and Lagrange's Theorem implies that $r$ divides $\left|\Omega_{4}^{-}(q)\right|=q^{2}\left(q^{4}-1\right)$ since $x$ fixes the decomposition $\tilde{V}=W \oplus W^{\perp}$ and acts trivially on $W$. If $r=2$ then the proof of $[6,2.7]$ implies that $x$ acts on $W^{\perp}$ as an $a_{2}$-involution, a contradiction since there are no such involutions in $\mathrm{O}_{4}^{-}(q)$. Now assume $r$ is odd. Let $i \geqslant 1$ be minimal such that $r$ divides $q^{i}-1$, so $i \in\{1,2,4\}$. We can rule out $i=4$ since $G_{0\left\langle v_{1}\right\rangle}=G_{2}(q)$ and $q^{2}+1$ does not divide $\left|G_{2}(q)\right|$. If $i \in\{1,2\}$ then $[\mathbf{4}, 3.29]$ implies that $\nu(x)=2$ (with respect to $\tilde{V}$ ) but it is clear from the proof of $[6,2.7]$ that there are no such elements in $\rho\left(G_{0}\right)$. We conclude that $b(G) \leqslant 4$.

It remains to show that $\bigcap_{i} G_{\left\langle v_{i}\right\rangle}$ is non-trivial for any non-singular vectors $v_{1}, v_{2}$ and $v_{3}$ which span a 3-dimensional subspace $U$ of $\tilde{V}$. If $x \in G_{0\left\langle v_{1}\right\rangle}=G_{2}(q)$ is a long root involution then $x$ lies in the $a_{2}$-class of $G$ (see $[6,2.7,2.13]$ ) and thus some conjugate $y$ of $x$ fixes the decomposition $\tilde{V}=U \oplus U^{\perp}$, acting trivially on $U$ and as an $a_{2}$-involution on $U^{\perp}$. We conclude that $y \in \bigcap_{i} G_{\left\langle v_{i}\right\rangle}$.

Lemma 3.2. Suppose $G_{0}=\operatorname{PSp}_{8}(q)$ and $H$ is of type $\operatorname{Sp}_{4}(q)$ 2 $S_{2}$ or $\operatorname{Sp}_{4}\left(q^{2}\right)$. Then $b(G)=3$.

Proof. If $q<4$ then the claim is easily checked using the computer package GAP so we will assume $q \geqslant 4$. Let $\bar{G}$ be a simple algebraic group over $\overline{\mathbb{F}}_{q}$ which admits a Frobenius morphism $\sigma$ such that $\bar{G}_{\sigma}$ has socle $G_{0}$. As before, let $x_{1}, \ldots, x_{k}$ represent the $G$-classes of elements of prime order in $H$ and let $Q(G, c)$ be the probability that a randomly chosen $c$-tuple of points in $\Omega$ does not form a base. Now Lemma 2.5 implies that $b(G) \geqslant 3$ and according to $(2.1)$ and (2.2) we have

$$
Q(G, c) \leqslant \widehat{Q}(G, c):=\sum_{i=1}^{k}\left|x_{i}^{G}\right| \cdot \operatorname{fpr}\left(x_{i}, \Omega\right)^{c}=\sum_{i=1}^{k}\left|x_{i}^{G}\right| \cdot\left(\left|x_{i}^{G} \cap H\right| /\left|x_{i}^{G}\right|\right)^{c} .
$$

(Note that we need only sum over $G$-classes of elements in $H$ since $\operatorname{fpr}(x, \Omega)=0$ if $x^{G} \cap H$ is empty.) Therefore, it suffices to show that there exists a function $F(q)$ such that $\widehat{Q}(G, 3) \leqslant$ $F(q)<1$ for all $q \geqslant 4$.

To avoid unnecessary repetition, we will assume $H$ is of type $\operatorname{Sp}_{4}(q) \prec S_{2}$ since a very similar argument applies when $H$ is of type $\operatorname{Sp}_{4}\left(q^{2}\right)$. We proceed by inspecting the proof of $[\mathbf{5}, 2.8]$. (To deal with the case $\mathrm{Sp}_{4}\left(q^{2}\right)$, the reader should consult the proof of [5, 3.3].) First suppose $x \in H \cap \operatorname{PGL}(V)$ is a semisimple element of odd prime order $r$ and let $i \geqslant 1$ be minimal such that $r$ divides $q^{i}-1$, so $i \in\{1,2,4\}$ and we consider each possibility for $i$ in turn. We note that $\left|x^{G}\right| \geqslant\left|x^{\bar{G}_{\sigma}}\right|$ by $[\mathbf{1 3}, 4.2 .2(\mathrm{j})]$. If $i=4$ then $r$ divides $q^{2}+1$ and $C_{G}(x)$ is of type $\mathrm{GU}_{2}\left(q^{2}\right)$, 
$\mathrm{Sp}_{4}(q) \times \mathrm{GU}_{1}\left(q^{2}\right)$ or $\mathrm{GU}_{1}\left(q^{2}\right)^{2}$ (see $[4,3.30]$, for example). Now, if $C_{G}(x)$ is of type $\mathrm{GU}_{2}\left(q^{2}\right)$ then

$$
\begin{gathered}
\left|x^{\bar{G}_{\sigma}} \cap H\right|=\left(\frac{\left|\operatorname{Sp}_{4}(q)\right|}{\left|\operatorname{GU}_{1}\left(q^{2}\right)\right|}\right)^{2}=q^{8}\left(q^{2}-1\right)^{4}, \\
\left|x^{\bar{G}_{\sigma}}\right|=\frac{\left|\operatorname{Sp}_{8}(q)\right|}{\left|\operatorname{GU}_{2}\left(q^{2}\right)\right|}=q^{14}\left(q^{2}-1\right)^{2}\left(q^{4}+1\right)\left(q^{6}-1\right)
\end{gathered}
$$

and there are precisely $\frac{1}{4}(r-1) \leqslant \frac{1}{4} q^{2}$ distinct $\bar{G}_{\sigma}$-classes of such elements. We also note that there are fewer than $\log \left(q^{2}+1\right)$ distinct odd prime divisors of $q^{2}+1$. In this way we deduce that the contribution to $\widehat{Q}(G, 3)$ from semisimple elements of odd prime order dividing $q^{2}+1$ is at most $\sum_{j=1}^{3} n_{j} b_{j}\left(a_{j} / b_{j}\right)^{3}$, where

\begin{tabular}{llll}
\hline$j$ & $n_{j}$ & $a_{j}$ & $b_{j}$ \\
\hline 1 & $\frac{1}{4} q^{2} \log \left(q^{2}+1\right)$ & $q^{8}\left(q^{2}-1\right)^{4}$ & $q^{14}\left(q^{2}-1\right)^{2}\left(q^{4}+1\right)\left(q^{6}-1\right)$ \\
2 & $\frac{1}{4} q^{2} \log \left(q^{2}+1\right)$ & $2 q^{4}\left(q^{2}-1\right)^{2}$ & $q^{12}\left(q^{2}-1\right)\left(q^{4}+1\right)\left(q^{6}-1\right)$ \\
3 & $\frac{1}{32} q^{4} \log \left(q^{2}+1\right)$ & $2 q^{8}\left(q^{2}-1\right)^{4}$ & $q^{16}\left(q^{2}-1\right)^{3}\left(q^{4}+1\right)\left(q^{6}-1\right)$ \\
\hline
\end{tabular}

The contribution from elements of odd prime order dividing $q^{2}-1$ can be estimated in a similar fashion. For example, if $C_{G}(x)$ is of type $\mathrm{GL}_{2}^{\epsilon}(q)^{2}$ then $r$ divides $q-\epsilon$,

$$
\left|x^{\bar{G}_{\sigma}} \cap H\right|=2\left(\frac{\left|\mathrm{Sp}_{4}(q)\right|}{\left|\mathrm{Sp}_{2}(q)\right|\left|\mathrm{GL}_{1}^{\epsilon}(q)\right|}\right)^{2}+\left(\frac{\left|\mathrm{Sp}_{4}(q)\right|}{\left|\mathrm{GL}_{1}^{\epsilon}(q)^{2}\right|}\right)^{2}=a_{4}, \quad\left|x^{\bar{G}_{\sigma}}\right|=\frac{\left|\mathrm{Sp}_{8}(q)\right|}{\left|\mathrm{GL}_{2}^{\epsilon}(q)^{2}\right|}=b_{4}
$$

and there are precisely $\left(\begin{array}{c}(r-1) / 2 \\ 2\end{array}\right)<\frac{1}{8} q^{2}$ distinct $\bar{G}_{\sigma}$-classes of such elements for each fixed prime $r$. Since there are fewer than $\log (q-\epsilon)$ odd prime divisors of $q-\epsilon$ we conclude that the contribution to $\widehat{Q}(G, 3)$ from these elements is at most $n_{4} b_{4}\left(a_{4} / b_{4}\right)^{3}$, where $n_{4}=\frac{1}{8} q^{2} \log (q-\epsilon)$. We leave the reader to examine the other possibilities for $C_{G}(x)$.

Next we consider unipotent elements. If $p>2$ then Lemma 2.4 and $[4,3.18,3.20]$ imply that the contribution to $\widehat{Q}(G, 3)$ from unipotent elements of order $p$ is at most $\sum_{j=1}^{6} d_{j}\left(c_{j} / d_{j}\right)^{3}$, where the terms $c_{j}$ and $d_{j}$ are defined as follows:

\begin{tabular}{llll}
\hline$j$ & $\lambda$ & $c_{j}$ & $d_{j}$ \\
\hline 1 & $\left(2,1^{6}\right)$ & $2\left(q^{4}-1\right)$ & $\frac{1}{2}\left(q^{8}-1\right)$ \\
2 & $\left(2^{2}, 1^{4}\right)$ & $2 q^{2}\left(q^{4}-1\right)+\left(q^{4}-1\right)^{2}$ & $\frac{1}{2} q\left(q^{2}-q+1\right)\left(q^{3}-1\right)\left(q^{8}-1\right)$ \\
3 & $\left(2^{3}, 1^{2}\right)$ & $2 q^{2}\left(q^{4}-1\right)^{2}$ & $\frac{1}{2} q^{2}\left(q^{2}+1\right)\left(q^{6}-1\right)\left(q^{8}-1\right)$ \\
4 & $\left(2^{4}\right)$ & $q^{4}\left(q^{4}-1\right)^{2}$ & $\frac{1}{2} q^{4}\left(q^{2}-1\right)\left(q^{6}-1\right)\left(q^{8}-1\right)$ \\
5 & $\left(4,1^{4}\right)$ & $2 q^{2}\left(q^{2}-1\right)\left(q^{4}-1\right)$ & $\frac{1}{2} q^{6}\left(q^{6}-1\right)\left(q^{8}-1\right)$ \\
6 & $\left(4^{2}\right)$ & $q^{4}\left(q^{2}-1\right)^{2}\left(q^{4}-1\right)^{2}$ & $\frac{1}{2} q^{9}(q-1)\left(q^{4}-1\right)\left(q^{6}-1\right)\left(q^{8}-1\right)$ \\
\hline
\end{tabular}

In the second column we list the partitions of 8 which correspond to the possible Jordan normal forms on $V$ of unipotent elements in $H$. The entries in the table are easily verified. For example, suppose $x \in H$ has corresponding partition $\lambda=\left(2^{2}, 1^{4}\right)$. Now, $\lambda$ can be written as a sum of 
two partitions of 4 in essentially two distinct ways, namely $\left(2^{2}\right) \oplus\left(1^{4}\right)$ and $\left(2,1^{2}\right) \oplus\left(2,1^{2}\right)$, and applying $[\mathbf{4}, 3.18]$ we deduce that

$$
\left|x^{G} \cap H\right| \leqslant 2 \frac{\left|\operatorname{Sp}_{4}(q)\right|}{\left|\mathrm{SO}_{2}^{+}(q)\right| q^{3}}+\left(\frac{\left|\mathrm{Sp}_{4}(q)\right|}{\left|\operatorname{Sp}_{2}(q)\right| q^{3}}\right)^{2}=c_{2}, \quad\left|x^{G}\right| \geqslant \frac{\left|\operatorname{Sp}_{8}(q)\right|}{\left|\operatorname{Sp}_{4}(q)\right|\left|\mathrm{O}_{2}^{-}(q)\right| q^{11}}=d_{2} .
$$

We can estimate the contribution from semisimple and unipotent involutions in the same way.

Finally, suppose $x$ is a field automorphism of prime order $r$. Then $r$ divides $\log _{p} q$ and the proof of $[\mathbf{5}, 2.8]$ gives $\left|x^{\bar{G}_{\sigma}} \cap H\right|<4 q^{20\left(1-\frac{1}{r}\right)}$ and $\left|x^{G}\right|>\frac{1}{2 d} q^{36\left(1-\frac{1}{r}\right)}=f(r, q)$, where $d=(2, q-1)$. Therefore $\operatorname{fpr}(x, \Omega)<8 d q^{-16\left(1-\frac{1}{r}\right)}=g(r, q)$ and so Lemma 2.4 implies that the contribution to $\widehat{Q}(G, 3)$ from field automorphisms is less than

$$
\sum_{r \in \pi}(r-1) \cdot h(r, q)<h(2, q)+2 h(3, q)+4 h(5, q)+\log _{p} q \cdot q^{36} g(7, q)^{3},
$$

where $h(r, q)=f(r, q) g(r, q)^{3}$ and $\pi$ is the set of distinct prime divisors of $\log _{p} q$.

In this way we obtain a function $F(q)$ which satisfies $\widehat{Q}(G, 3) \leqslant F(q)<1$ for all $q \geqslant 4$. We conclude that $b(G)=3$ for all values of $q$.

Proposition 3.3. If $G_{0}=\operatorname{PSp}_{n}(q)$ and $n \geqslant 6$ then $b(G) \leqslant 4$.

Proof. By applying Propositions 2.2 and 2.3 we see that $b(G) \leqslant c$ if

$$
c(1 / 2-1 / n-\iota)-1 \geqslant 1 / 3 .
$$

In particular, if $\iota=0$ then $b(G) \leqslant 4$ for all $n \geqslant 6$ so assume $\iota>0$. If $\iota \in\{1 / n, 1 /(n+2)\}$ then $n \equiv 0(4)$ and $H$ is of type $\operatorname{Sp}_{n / 2}(q) \imath S_{2}$ or $\operatorname{Sp}_{n / 2}\left(q^{2}\right)$ (see [3, Table 1]). Here (3.2) implies that $b(G) \leqslant 4$ if $n \geqslant 12$, while Lemma 3.2 applies if $n=8$. According to [3, Table 1] it remains to deal with the following cases:

\begin{tabular}{lllll}
\hline & $G_{0}$ & type of $H$ & $\iota$ & $b(G)$ \\
\hline (i) & $\mathrm{Sp}_{8}(2)$ & $A_{10}$ & .062 & 3 \\
(ii) & $\mathrm{Sp}_{6}(q)$ & $G_{2}(q), p=2$ & .084 & 4 \\
(iii) & $\mathrm{Sp}_{6}(2)$ & $\mathrm{U}_{3}(3)$ & .054 & 4 \\
\hline
\end{tabular}

In (i) and (iii) we use GAP $[\mathbf{1 1}]$ to calculate $b(G)$. See Lemma 3.1 for (ii).

3.2. $G_{0}=\mathrm{P} \Omega_{n}^{\epsilon}(q)$

Now suppose $G_{0}$ is an orthogonal group. Note that we may assume $n \geqslant 7$ and that $q$ is odd if $n$ is odd.

Lemma 3.4. Suppose $G_{0}=\mathrm{P} \Omega_{8}^{+}(q)$ and $H$ is of type $\mathrm{GL}_{4}^{\epsilon^{\prime}}(q)$, or $G_{0}=\mathrm{P} \Omega_{10}^{\epsilon}(q)$ and $H$ is of type $\mathrm{GL}_{5}^{\epsilon^{\prime}}(q)$. Then $b(G) \in\{3,4\}$. 
Proof. First note that Lemma 2.5 gives $b(G) \geqslant 3$. For the upper bound we proceed as in Lemma 3.2: close inspection of the proof of $[\mathbf{5}, 3.3]$ yields a function $F(q)$ which satisfies $\widehat{Q}(G, 4) \leqslant F(q)<1$ for all values of $q$, where $\widehat{Q}(G, 4)$ is defined as in (3.1). This implies that $b(G) \leqslant 4$. The details are left to the reader.

Proposition 3.5. If $G_{0}=\mathrm{P} \Omega_{n}^{\epsilon}(q)$ and $n \geqslant 7$ then $b(G) \leqslant 4$.

Proof. If $\iota=0$ then (3.2) implies that $b(G) \leqslant 4$ so assume $\iota>0$. If $H$ is of type $\mathrm{GL}_{n / 2}^{\epsilon^{\prime}}(q)$ then $\iota=1 /(n-2)$ and the conclusion $b(G) \leqslant 4$ follows via Proposition 2.2 and Remark 4 if $n \geqslant 12$; the cases $n \in\{8,10\}$ were considered in Lemma 3.4 (note that we may assume $\epsilon=+$ if $n=8$ - see [20, Table 3.5.F], for example). According to [3, Table 1] it remains to deal with the cases listed in the next table. Here we omit the irreducible embedding $\Omega_{7}(q)<\mathrm{P} \Omega_{8}^{+}(q)$ since the corresponding action is equivalent to a subspace action - see Table 1.

\begin{tabular}{lllll}
\hline & $G_{0}$ & type of $H$ & $\iota$ & $b(G)$ \\
\hline (i) & $\Omega_{10}^{-}(2)$ & $A_{12}$ & .087 & $3+\alpha$ \\
(ii) & $\mathrm{P} \Omega_{8}^{+}(3)$ & $\Omega_{8}^{+}(2)$ & .081 & $3+\alpha$ \\
(iii) & $\Omega_{8}^{+}(2)$ & $\mathrm{O}_{4}^{-}(2) \imath S_{2}$ & .001 & 3 \\
(iv) & $\Omega_{8}^{+}(2)$ & $A_{9}$ & .124 & 4 \\
(v) & $\Omega_{7}(q)$ & $G_{2}(q), p>2$ & .108 & 4 \\
(vi) & $\Omega_{7}(3)$ & $\mathrm{Sp}_{6}(2)$ & .065 & 3 \\
\hline
\end{tabular}

The results for (i)-(iv) and (vi) can be checked using GAP $[\mathbf{1 1}]$; here $\alpha=0$ if $G=G_{0}$, otherwise $\alpha=1$.

For (v) we argue as in the proof of Lemma 3.1. Here $G=G_{0} \cdot\langle\phi\rangle$ and $H=G_{2}(q) \cdot\langle\phi\rangle$, where $\phi$ is a (possibly trivial) field automorphism of $G_{0}$; the action of $G$ on $\Omega$ is equivalent to the action of $\rho\left(G_{0}\right) \cdot\langle\phi\rangle$ on the set of 1-dimensional non-singular subspaces of the natural module $\tilde{V}$ for $J$, where $\rho$ is an irreducible spin representation of $J=\mathrm{P}_{8}^{+}(q)$. Seeking a contradiction, suppose $x \in \bigcap_{i=1}^{4} G_{\left\langle v_{i}\right\rangle}$ has prime order $r$, where the $v_{i}$ are non-singular vectors which span a - 4-subspace $W$ of $\tilde{V}$ and have the property that the subspaces $\left\langle v_{i}\right\rangle$ are not simultaneously fixed by a field automorphism of $G_{0}$. Then $x$ fixes the decomposition $\tilde{V}=W \oplus W^{\perp}$ and acts trivially on $W$. If $r>2$ we get a contradiction precisely as in the proof of Lemma 3.1 so we may assume $r=2$. Then $[4,3.55]$ implies that $C_{J}(x)$ is of type $\mathrm{GL}_{4}^{\epsilon}(q), \mathrm{O}_{4}^{+}(q)^{2}$ or $\mathrm{O}_{4}^{+}\left(q^{2}\right)$, and this contradicts the fact that $x$ fixes the above decomposition and acts trivially on $W$. We conclude that $b(G) \leqslant 4$. To see that equality holds, let $v_{1}, v_{2}$ and $v_{3}$ be any non-singular vectors which span a 3 -dimensional subspace $U$ of $\tilde{V}$ and let $x \in G_{0\left\langle v_{1}\right\rangle}=G_{2}(q)$ be a long root element. Then $x$ has order $p$ and the proof of $[6,2.13]$ implies that $x$ has Jordan form $\left[J_{2}^{2}, J_{1}^{4}\right]$ 
on $\tilde{V}$, where $J_{i}$ denotes a standard Jordan block of size $i$. In particular, some conjugate $y$ of $x$ fixes the decomposition $\tilde{V}=U \oplus U^{\perp}$ and acts trivially on $U$. This implies that $y \in \bigcap_{i} G_{\left\langle v_{i}\right\rangle}$ and thus $b(G)>3$.

3.3. $G_{0}=\operatorname{PSL}_{n}^{\epsilon}(q)$

We begin with the exceptional case referred to in the statement of Theorem 1.

Lemma 3.6. Suppose $G_{0}=\mathrm{U}_{6}(2)$ and $H$ is an irreducible subgroup of type $\mathrm{U}_{4}(3)$. Then $b(G)=5$ if $G=G_{0} .2$, otherwise $b(G)=4$.

Proof. With the aid of the Online Atlas of Finite Groups, one can construct $G$ and $H$ in GAP and the desired result is easily checked.

Lemma 3.7. Suppose $G_{0}=\operatorname{PSL}_{n}^{\epsilon}(q)$ and $H$ is of type $\operatorname{Sp}_{n}(q)$, where $n \in\{6,8\}$. Then $b(G) \in\{3,4\}$.

Proof. First observe that Lemma 2.5 implies that $b(G) \geqslant 3$. If $(n, q)=(6,2)$ then using GAP [11] we calculate that $b(G)=3+\alpha$, where $\alpha=1$ if $G=\mathrm{U}_{6}(2) .2$, otherwise $\alpha=0$. For $(n, q) \neq(6,2)$ we proceed as in Lemma 3.2 ; by inspecting the proof of $[4,8.1]$ we derive a function $F(q)$ such that $\widehat{Q}(G, 4) \leqslant F(q)<1$ for all values of $q$.

Proposition 3.8. If $G_{0}=\operatorname{PSL}_{n}^{\epsilon}(q)$ and $n \geqslant 6$ then $b(G) \leqslant 4$.

Proof. As before, we quickly reduce to the case $\iota>0$. Suppose $H$ is of type $\operatorname{Sp}_{n}(q)$, in which case $\iota=1 / n$. Now, if $n \geqslant 12$ then (3.2) holds with $c=4$; the case $n=10$ follows from Proposition 2.2 since $T_{G}<4 / 15$ (see Remark 4), while the cases $n \in\{6,8\}$ are handled in Lemma 3.7. According to [3, Table 1], it remains to deal with the case $G_{0}=\mathrm{U}_{6}(2)$ with $H$ an irreducible subgroup of type $\mathrm{U}_{4}(3)$. This is the content of Lemma 3.6 above.

\section{Proof of Theorem 1, Part II: $n<6$}

In this final section we complete the proof of Theorem 1 by dealing with the remaining groups of small rank. Here Theorem 2.1 is less useful and we proceed by considering each maximal non-subspace subgroup of $G$ in turn. As before, we use the proof of $[\mathbf{3}$, Theorem 1] in $[\mathbf{4}, \mathbf{5}$, 6] and the bounds on fixed point ratios therein. Again, the computer package GAP is a useful tool when the underlying field is small. 
Our detailed results are recorded in Tables 5 and 6 . The maximal non-subspace subgroups are

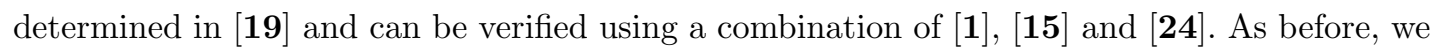
exclude any subgroup for which the corresponding primitive action is equivalent to a standard action of an isomorphic group (see Table 1). The third column lists certain necessary, but not always sufficient, conditions for the corresponding action to be primitive and non-standard. In the column headed ' $b(G)$ ' we record the possibilities for $b(G)$. Where more than one possibility is listed, we mean that $b(G)$ always takes one of the listed values, but we do not claim that each possibility is attainable.

The next proposition follows from the results in Tables 5 and 6 and completes the proof of Theorem 1. In Table 2 we adopt the notation of [10] for labelling representatives of $G_{0}$-classes of involutions when $G_{0}=\mathrm{U}_{4}(3)$.

Proposition 4.1. Let $G$ be a finite almost simple group with socle $G_{0}$, where $G_{0}=$ $\operatorname{PSp}_{4}(q)^{\prime}$ or $\operatorname{PSL}_{n}^{\epsilon}(q)$ with $n \leqslant 5$. If $\Omega$ is a faithful primitive non-standard $G$-set then either $b(G) \leqslant 3$, or $b(G)=4$ and $(G, H)$ is listed in Table 2 , where $H=G_{\omega}$.

The cases in Table 2 can be checked using GAP. For the remainder, we use the proof of $[\mathbf{3}$, Theorem 1] to define a function $F(q)$ such that $\widehat{Q}(G, c) \leqslant F(q)<1$ (see (3.1)), with $c=2$ or 3 as required. For small values of $q$, precise base size calculations are often possible using GAP. Below we provide some details when $G_{0}=\operatorname{PSp}_{4}(q)^{\prime}$ and $\mathrm{L}_{2}(q)$. (Note that the case $G_{0}=\mathrm{L}_{2}(q)$ was not considered in [3] since Theorem 2.1 is trivial in this case.)

\section{1. $G_{0}=\operatorname{PSp}_{4}(q)^{\prime}$}

Let $G$ be a finite almost simple group with socle $G_{0}=\operatorname{PSp}_{4}(q)^{\prime}$. We use the symbol $(\leftrightarrow)$ to denote the additional hypothesis " $p=2$ and $G$ contains graph-field automorphisms". (Note that some authors favour the term graph automorphism here.)

\begin{tabular}{lll}
\hline$G_{0}$ & type of $H$ & remarks \\
\hline $\mathrm{U}_{4}(3)$ & $2^{4} \cdot \operatorname{Sp}_{4}(3)$ & $G=G_{0} \cdot\langle 2 B, 2 D\rangle$ \\
& $\mathrm{L}_{3}(4)$ & \\
$\mathrm{Sp}_{4}(2)^{\prime}$ & $\mathrm{O}_{2}^{-}(2) \imath S_{2}$ & $G=\operatorname{Aut}\left(G_{0}\right)$ \\
$\mathrm{U}_{3}(3)$ & $\mathrm{L}_{3}(2)$ & $G=G_{0} .2$ \\
\hline
\end{tabular}

TABLE 2. $n<6$ : The cases with $b(G)=4$ 
Proposition 4.2. Let $G$ be a finite almost simple group with socle $G_{0}=\operatorname{PSp}_{4}(q)^{\prime}$ and let $\Omega$ be a faithful primitive non-standard $G$-set. Then the possibilities for $H=G_{\omega}$ are listed in Table 3 .

Proof. The maximal subgroups of $G$ are listed in [19, Chapter 5] and [1, 14.2] (also see [20, Table 3.5.C]). The subgroups in Aschbacher's $\mathscr{C}_{1}$ collection can be omitted since they are subspace subgroups. In addition, each action corresponding to a subgroup of type $\operatorname{Sp}_{2}(q)$ 乙 $S_{2}$, $\mathrm{Sp}_{2}\left(q^{2}\right)$ or $2^{4} \cdot \mathrm{O}_{4}^{-}(2)(q=3)$ is equivalent to a $\mathscr{C}_{1}$-action of an isomorphic classical group and so these subgroups are excluded too (see Table 1). The second column records necessary conditions for the relevant action to be primitive and non-standard.

Lemma 4.3. If $H$ is of type $2^{4}$. $\mathrm{O}_{4}^{-}(2)$ then $b(G)=3$ if $G=\mathrm{PGSp}_{4}(5)$, otherwise $b(G)=2$.

Proof. Here $q=p>3$ and $H \leqslant 2^{4} . \mathrm{O}_{4}^{-}(2)=\widetilde{H}$ is a subgroup in Aschbacher's $\mathscr{C}_{6}$ collection. If $q<17$ then the result can be checked using GAP so we will assume $q \geqslant 17$. Now the prime divisors of $|\widetilde{H}|$ are 2,3 and 5 , and the proof of $[4,6.6]$ gives $i_{r}(\widetilde{H}) \leqslant n_{r}$, where $n_{5}=384$, $n_{3}=80$ and $n_{2}=155$, and $i_{r}(\widetilde{H})$ is the number of elements of order $r$ in $\widetilde{H}$. Therefore $\widehat{Q}(G, 2) \leqslant\left(384^{2}+80^{2}\right) a_{1}^{-1}+155^{2} a_{2}^{-1}$, where

$$
a_{1}=\frac{\left|\mathrm{Sp}_{4}(q)\right|}{\left|\mathrm{GU}_{2}(q)\right|}=q^{3}(q-1)\left(q^{2}+1\right), \quad a_{2}=\frac{\left|\mathrm{Sp}_{4}(q)\right|}{\left|\operatorname{Sp}_{2}\left(q^{2}\right)\right| 2}=\frac{1}{2} q^{2}\left(q^{2}-1\right),
$$

and thus $\widehat{Q}(G, 2)<1$ for all $q \geqslant 17$.

Lemma 4.4. If $H$ is of type $S z(q)$ then $b(G)=3$.

\begin{tabular}{ll}
\hline type of $H$ & conditions \\
\hline $\mathrm{GL}_{2}(q) \cdot 2$ & $q$ odd \\
$\mathrm{GU}_{2}(q)$ & $q$ odd \\
$\mathrm{Sp}_{4}\left(q_{0}\right)$ & $q=q_{0}^{k}, k$ prime \\
$2^{4} \cdot \mathrm{O}_{4}^{-}(2)$ & $q=p>3$ \\
$S z(q)$ & $p=2, \log _{2} q>1$ odd \\
$\mathrm{L}_{2}(q)$ & $p>3$ \\
$A_{6}$ & $q=p>3$ \\
$A_{7}$ & $q=7$ \\
$\mathrm{O}_{2}^{\epsilon}(q) \imath S_{2}$ & $(\leftrightarrow)$ holds, $\epsilon=-$ if $q=2$ \\
$\mathrm{O}_{2}^{-}\left(q^{2}\right) \cdot 2$ & $(\leftrightarrow)$ holds \\
\hline
\end{tabular}

TABLE 3. $G_{0}=\operatorname{PSp}_{4}(q)^{\prime}$ : Maximal non-subspace subgroups 
Proof. Here $q$ is even, $\log _{2} q>1$ is odd and $H \cap \operatorname{PGL}(V)=S z(q)$ is the centralizer in $G_{0}$ of an involutory graph-field automorphism, where $V$ is the natural $G_{0}$-module. Now, if $G \neq G_{0}$ then

$$
\frac{\log |G|}{\log |\Omega|} \geqslant \frac{\log \left(2 q^{4}\left(q^{2}-1\right)\left(q^{4}-1\right)\right)}{\log \left(q^{2}(q+1)\left(q^{2}-1\right)\right)}>2
$$

for all $q \geqslant 8$ and thus Lemma 2.5 implies that $b(G) \geqslant 3$. The case $G=G_{0}$ is studied in [21]; the subdegrees of $G$ are listed in [21, Table 1] and we immediately deduce that $b(G) \geqslant 3$. Therefore, it suffices to show that there exists a function $F(q)$ such that $\widehat{Q}(G, 3) \leqslant F(q)<1$ for all values of $q$.

Suppose $x \in H \cap \operatorname{PGL}(V)$ has prime order $r$. If $r=2$ then $x$ is $G$-conjugate to $c_{2}$ (see [2] and $[4,2.56])$ and thus

$$
\left|x^{G} \cap H\right|=\left(q^{2}+1\right)(q-1)=a_{1}, \quad\left|x^{G}\right|=\left(q^{2}-1\right)\left(q^{4}-1\right)=b_{1} .
$$

Now assume $r>2$. Then $[4,2.56]$ implies that $r \geqslant 5, \nu(x)=3$ (see Definition 4) and $i \in\{1,4\}$, where $i \geqslant 1$ is minimal such that $r$ divides $q^{i}-1$. If $i=1$ then $r \geqslant 7$ and $[4,2.56]$ gives

$$
\left|x^{\mathrm{Sp}_{4}(q)} \cap H\right| \leqslant \frac{1}{r}|S z(q)|=\frac{1}{r} a_{2}, \quad\left|x^{\mathrm{Sp}_{4}(q)}\right|=\left|\mathrm{Sp}_{4}(q): \mathrm{GL}_{1}(q)^{2}\right|=b_{2} .
$$

In addition, there are precisely $\frac{1}{2}(r-1)$ possibilities for $x$ up to $\operatorname{Sp}_{4}(q)$-conjugacy and so the contribution to $\widehat{Q}(G, 3)$ from semisimple elements whose order divides $q-1$ is at most

$$
b_{2}\left(a_{2} / b_{2}\right)^{3} \frac{1}{2} \sum_{r \in \Lambda} \frac{r-1}{r^{3}},
$$

where $\Lambda$ is the set of distinct prime divisors of $q-1$. Now

$$
\frac{1}{2} \sum_{r \in \Lambda} \frac{r-1}{r^{3}}<\frac{1}{2} \sum_{m=3}^{\infty}\left(\frac{1}{2 m+1}\right)^{2}=\frac{1}{16} \pi^{2}-\frac{1}{2}-\frac{1}{18}-\frac{1}{50}=n_{2}
$$

and so the contribution here is less than $n_{2} b_{2}\left(a_{2} / b_{2}\right)^{3}$. Similarly, the elements of prime order dividing $q^{2}+1$ contribute less than $n_{3} b_{3}\left(a_{3} / b_{3}\right)^{3}$, where

$$
a_{3}=q^{2}\left(q^{2}+1\right)(q-1), \quad b_{3}=q^{4}\left(q^{2}-1\right)^{2}, \quad n_{3}=\frac{1}{32} \pi^{2}-\frac{1}{4}-\frac{1}{36} .
$$

Now, if $x \in G$ is an involutory graph-field automorphism then we may assume $x$ centralizes $H \cap \operatorname{PGL}(V)$ and thus

$$
\left|x^{G} \cap H\right|=i_{2}(S z(q))+1=\left(q^{2}+1\right)(q-1)+1=a_{4}, \quad\left|x^{G}\right|=q^{2}(q+1)\left(q^{2}-1\right)=b_{4} .
$$

Finally, if $x$ is a field automorphism of prime order $r$ then

$$
\left|x^{G}\right|=\frac{q^{4}\left(q^{2}-1\right)\left(q^{4}-1\right)}{q^{\frac{4}{r}}\left(q^{\frac{2}{r}}-1\right)\left(q^{\frac{4}{r}}-1\right)}=f(r, q), \quad f p r(x, \Omega)=\frac{q^{\frac{2}{r}}\left(q^{\frac{1}{r}}+1\right)\left(q^{\frac{2}{r}}-1\right)}{q^{2}(q+1)\left(q^{2}-1\right)}=g(r, q)
$$


and so the contribution here is at most

$$
\sum_{r \in \Gamma}(r-1) \cdot h(r, q)<2 h(3, q)+\log _{2} q \cdot q^{4}\left(q^{2}-1\right)\left(q^{4}-1\right) \cdot g(5, q)^{3}=F_{1}(q),
$$

where $h(r, q)=f(r, q) g(r, q)^{3}$ and $\Gamma$ is the set of distinct prime divisors of $\log _{2} q$. We conclude that

$$
\widehat{Q}(G, 3)<\sum_{j=1}^{4} n_{j} b_{j}\left(a_{j} / b_{j}\right)^{3}+F_{1}(q)=F(q)
$$

(where $n_{1}=n_{4}=1$ ) and one can check that $F(q)<1$ for all $q \geqslant 8$.

The remaining cases in Table 3 are dealt with in a similar fashion and we present our results in Table 6.

\section{2. $G_{0}=\mathrm{L}_{2}(q)$}

Finally, suppose $G$ is an almost simple group with socle $G_{0}=\mathrm{L}_{2}(q)$. Note that we may assume $q>4$ since $\mathrm{L}_{2}(2) \cong S_{3}, \mathrm{~L}_{2}(3) \cong A_{4}$ and $\mathrm{L}_{2}(4) \cong \mathrm{L}_{2}(5)$. The maximal subgroups of $G$ are well-known; in Table 4 we list the ones which correspond to faithful non-standard actions.

Lemma 4.5. If $H$ is of type $\mathrm{GL}_{1}\left(q^{2}\right)$ then $b(G) \in\{2,3\}$.

Proof. If $q=5$ then a calculation with GAP gives $b(G)=3$ if $G=\operatorname{PGL}_{2}(5)$, otherwise $b(G)=2$. For the remainder let us assume $q \geqslant 7$. Let $V$ denote the natural $G_{0}$-module and observe that $H \cap \operatorname{PGL}(V) \leqslant C_{q+1} .2 \cong D_{2(q+1)}$. Define $\widehat{Q}(G, 3)$ as in (3.1) and let $x \in H \cap$ $\operatorname{PGL}(V)$ be an element of prime order $r$. If $r=p$ then $p=2$ and we have $\left|x^{G} \cap H\right|=q+1=a_{1}$ and $\left|x^{G}\right|=q^{2}-1=b_{1}$. Next assume $r \neq p$. If $r=2$ and $C_{G}(x)$ is of type $\mathrm{GL}_{1}(q)^{2}$ then $\left|x^{G} \cap H\right|=\frac{1}{2}(q+1)=a_{2}$ and $\left|x^{G}\right|=\frac{1}{2} q(q+1)=b_{2}$, otherwise $C_{G}(x)$ is of type $\operatorname{GL}_{1}\left(q^{2}\right)$ and we have $\left|x^{G} \cap H\right|=\frac{1}{2}(q+3)=a_{3}$ and $\left|x^{G}\right|=\frac{1}{2} q(q-1)=b_{3}$. If $r \neq p$ is odd then $r$ divides $q+1$ and it is easy to see that $\left|x^{\tilde{G}} \cap H\right|=2=a_{4}$ and $\left|x^{\tilde{G}}\right|=q(q-1)$, where $\tilde{G}=\operatorname{PGL}_{2}(q)$. For such a prime $r$, there are precisely $\frac{1}{2}(r-1) \leqslant \frac{1}{2} q$ possibilities for $x$ up to $\tilde{G}$-conjugacy and we note

\begin{tabular}{ll}
\hline type of $H$ & conditions \\
\hline $\operatorname{GL}_{1}(q) \imath S_{2}$ & $q>5$ \\
$\operatorname{GL}_{1}\left(q^{2}\right)$ & \\
$\operatorname{GL}_{2}\left(q_{0}\right)$ & $q=q_{0}^{k}, k>2$ prime \\
$2^{2} \cdot \mathrm{O}_{2}^{-}(2)$ & $q=p>7$ \\
$A_{5}$ & $q \equiv \pm 1(10), q \neq 9$ \\
\hline
\end{tabular}

TABLE 4. $G_{0}=\mathrm{L}_{2}(q)$ : Maximal non-subspace subgroups 
that $q+1$ has fewer than $\log (q+1)$ odd prime divisors. We conclude that the contribution to $\widehat{Q}(G, 3)$ from $H \cap \operatorname{PGL}(V)$ is at most

$$
F_{1}(q)=\sum_{i=1}^{3} b_{i}\left(a_{i} / b_{i}\right)^{3}+n_{4} b_{4}\left(a_{4} / b_{4}\right)^{3},
$$

where $n_{4}=\frac{1}{2} q \log (q+1)$.

Finally, suppose $x \in H$ is a field automorphism of $G_{0}$ of prime order $r$. Here $q=q_{0}^{r}$ and we note that $r$ is odd since $x \in H-\operatorname{PGL}(V)$. Now $\left|x^{G} \cap H\right| \leqslant q+1$ since $x^{G} \cap H \subseteq C_{q+1} x$, and we have

$$
\left|x^{G}\right| \geqslant \frac{1}{d}\left|x^{\tilde{G}}\right|=\frac{1}{d} \frac{q\left(q^{2}-1\right)}{q^{\frac{1}{r}}\left(q^{\frac{2}{r}}-1\right)}=f(r, q),
$$

where $d=(2, q-1)$. Therefore $\operatorname{fpr}(x, \Omega) \leqslant(q+1) f(r, q)^{-1}=g(r, q)$ and thus

$$
\begin{aligned}
\widehat{Q}(G, 3) & \leqslant F_{1}(q)+\sum_{r \in \Lambda}(r-1) \cdot h(r, q) \\
& \leqslant F_{1}(q)+\alpha\left[2 h(3, q)+4 h(5, q)+\log _{p} q \cdot q\left(q^{2}-1\right) \cdot g(7, q)^{3}\right]=F(q),
\end{aligned}
$$

where $h(r, q)=f(r, q) g(r, q)^{3}$ and $\Lambda$ is the set of distinct odd primes which divide $\log _{p} q$. We set $\alpha=1$ if $\Lambda$ is non-empty, otherwise $\alpha=0$. The reader can check that $F(q)<1$ for all $q \geqslant 7$.

A similar argument applies when $H$ is of type $\mathrm{GL}_{1}(q) 2 S_{2}$ or $\mathrm{GL}_{2}\left(q_{0}\right)$ (note that in the former case we have $b(G)=2$ if $G \leqslant \mathrm{PGL}_{2}(q)$ - see $\left.[\mathbf{1 7}, 2.1]\right)$. It remains to consider the final two cases in Table 4.

Lemma 4.6. If $H$ is of type $2^{2} . \mathrm{O}_{2}^{-}(2)$ then $b(G)=2$.

Proof. First observe that $H \cap \operatorname{PGL}(V) \leqslant S_{4}$. According to Table 1, we may assume $q>7$. If $q<17$ then the result is easily checked via GAP so we will assume $q \geqslant 17$. Define $\widehat{Q}(G, c)$ as in (3.1) and let $x \in H$ be an element of prime order $r$. Then $\left|x^{G}\right| \geqslant 2^{-\delta_{2, r}} q(q-1)$ and thus

$$
\widehat{Q}(G, 2) \leqslant \frac{1}{2} q(q-1)\left(\frac{18}{q(q-1)}\right)^{2}+q(q-1)\left(\frac{8}{q(q-1)}\right)^{2}=F(q)
$$

since $i_{2}\left(S_{4}\right)=9$ and $i_{3}\left(S_{4}\right)=8$, where $i_{r}\left(S_{4}\right)$ is the number of elements of order $r$ in $S_{4}$. We conclude that $b(G)=2$ since $F(q)<1$ for all $q \geqslant 17$.

Lemma 4.7. If $H$ is of type $A_{5}$ then $b(G)=2+\delta_{11, q}+\delta_{19, q}$.

Proof. Here $q \leqslant p^{2}$ and the maximality of $H$ implies that $(G, H)=\left(G_{0}, A_{5}\right)$ if $q=p$. We will assume $q>49$ since the other cases can be checked using GAP (note that $q \neq 9$ - see Table 
1). Now, if $x \in H \cap \operatorname{PGL}(V)=A_{5}$ has prime order $r$ then $\left|x^{G}\right| \geqslant 2^{-\delta_{2, r}} q(q-1)$ and so Lemma 2.4 implies that the contribution to $\widehat{Q}(G, 2)$ from $H \cap \operatorname{PGL}(V)$ is at most

$$
F_{1}(q)=\frac{1}{2} q(q-1)\left(\frac{30}{q(q-1)}\right)^{2}+q(q-1)\left(\frac{20}{q(q-1)}\right)^{2}+2 q(q-1)\left(\frac{12}{q(q-1)}\right)^{2}
$$

since $i_{2}\left(A_{5}\right)=15, i_{3}\left(A_{5}\right)=20$ and $i_{5}\left(A_{5}\right)=12$. Finally, if $q=p^{2}$ and $x \in H-\operatorname{PGL}(V)$ is an involution then $\left|x^{G} \cap H\right|=10,\left|x^{G}\right| \geqslant \frac{1}{2} q^{1 / 2}(q+1)$ and thus

$$
\widehat{Q}(G, 2) \leqslant F_{1}(q)+\left(\log _{p} q-1\right) \cdot q^{\frac{1}{2}}(q+1) \cdot\left(\frac{20}{q^{1 / 2}(q+1)}\right)^{2}=F(q) .
$$

The reader can check that $F(q)<1$ for all $q \geqslant 49$. 


\begin{tabular}{|c|c|c|c|c|}
\hline$G_{0}$ & type of $H$ & conditions & $b(G)$ & remarks \\
\hline \multirow[t]{8}{*}{$\mathrm{L}_{5}(q)$} & $\mathrm{GL}_{1}(q) \prec S_{5}$ & $q>3$ & 2 & see $[\mathbf{1 7}]$ \\
\hline & $\mathrm{GL}_{1}\left(q^{5}\right)$ & & 2 & \\
\hline & $\mathrm{GL}_{5}\left(q_{0}\right)$ & $q=q_{0}^{k}, k$ prime & 2,3 & $b(G)=2$ if $k>2$ \\
\hline & $5^{2} \cdot \mathrm{Sp}_{2}(5)$ & $q=p \equiv 1(5)$ & 2 & \\
\hline & $\mathrm{O}_{5}(q)$ & $q$ odd & 2,3 & \\
\hline & $\mathrm{U}_{5}\left(q_{0}\right)$ & $q=q_{0}^{2}$ & 2,3 & \\
\hline & $\mathrm{L}_{2}(11)$ & $q=p \equiv 1,3,4,5,9(11)$ & 2 & \\
\hline & $M_{11}$ & $q=3$ & 2 & \\
\hline \multirow[t]{7}{*}{$\mathrm{U}_{5}(q)$} & $\mathrm{GU}_{1}(q) \imath S_{5}$ & & $2+\delta_{2, q}$ & \\
\hline & $\mathrm{GU}_{1}\left(q^{5}\right)$ & & 2 & \\
\hline & $\mathrm{GU}_{5}\left(q_{0}\right)$ & $q=q_{0}^{k}, k>2$ prime & 2 & \\
\hline & $5^{2} \cdot \mathrm{Sp}_{2}(5)$ & $p \equiv 2,3,4(5), \quad q \equiv 4(5)$ & 2 & \\
\hline & $\mathrm{O}_{5}(q)$ & $q$ odd & 2,3 & \\
\hline & $\mathrm{L}_{2}(11)$ & $q=p \equiv 2,6,7,8,10(11)$ & 2 & \\
\hline & $\mathrm{U}_{4}(2)$ & $q=5$ & 2 & \\
\hline \multirow[t]{10}{*}{$\mathrm{L}_{4}(q)$} & $\mathrm{GL}_{1}(q) \prec S_{4}$ & $q>3$ & 2 & see $[\mathbf{1 7}]$ \\
\hline & $\mathrm{GL}_{2}(q) \curlywedge S_{2}$ & $q>2$ & $3-\delta_{2, p}$ & see $[\mathbf{1 7}]$ \\
\hline & $\mathrm{GL}_{2}\left(q^{2}\right)$ & $q>2$ & 2,3 & $b(G)=3$ if $q=3$ \\
\hline & $\mathrm{GL}_{4}\left(q_{0}\right)$ & $q=q_{0}^{k}, k$ prime & 2,3 & $b(G)=2$ if $k>2$ \\
\hline & $2^{4} \cdot \mathrm{Sp}_{4}(2)$ & $q=p \equiv 1(4)$ & 2 & \\
\hline & $\mathrm{O}_{4}^{+}(q)$ & $q$ odd & 2,3 & \\
\hline & $\mathrm{O}_{4}^{-}(q)$ & $q$ odd & 2,3 & \\
\hline & $\mathrm{U}_{4}\left(q_{0}\right)$ & $q=q_{0}^{2}$ & 2,3 & $b(G)=3$ if $q=4$ \\
\hline & $A_{7}$ & $q=p \equiv 1,2,4(7), \quad q>2$ & 2 & \\
\hline & $\mathrm{U}_{4}(2)$ & $q=p \equiv 1(6)$ & 2 & \\
\hline \multirow[t]{10}{*}{$\mathrm{U}_{4}(q)$} & $\mathrm{GU}_{1}(q) \imath S_{4}$ & & 2,3 & $b(G)=2$ if $q>3$ \\
\hline & $\mathrm{GU}_{2}(q) \curlywedge S_{2}$ & & 2,3 & \\
\hline & $\mathrm{GL}_{2}\left(q^{2}\right)$ & & 2,3 & \\
\hline & $\mathrm{GU}_{4}\left(q_{0}\right)$ & $q=q_{0}^{k}, k>2$ prime & 2 & \\
\hline & $2^{4} . \mathrm{Sp}_{4}(2)$ & $q=p \equiv 3(4)$ & $2,3,4$ & $b(G)=2$ if $q>3$ \\
\hline & $\mathrm{O}_{4}^{+}(q)$ & $q$ odd & 2,3 & \\
\hline & $\mathrm{O}_{4}^{-}(q)$ & $q$ odd & 2,3 & \\
\hline & $A_{7}$ & $q=p \equiv 3,5,6(7)$ & $2+\delta_{3, q}$ & \\
\hline & $\mathrm{L}_{3}(4)$ & $q=3$ & 4 & \\
\hline & $\mathrm{U}_{4}(2)$ & $q=p \equiv 5(6)$ & 2 & \\
\hline
\end{tabular}

TABle 5. Base sizes for non-standard actions, I 


\begin{tabular}{|c|c|c|c|c|}
\hline$G_{0}$ & type of $H$ & conditions & $b(G)$ & remarks \\
\hline \multirow[t]{10}{*}{$\mathrm{PSp}_{4}(q)^{\prime}$} & $\mathrm{GL}_{2}(q) \cdot 2$ & $q$ odd & 2,3 & \\
\hline & $\mathrm{GU}_{2}(q)$ & $q$ odd & 2,3 & \\
\hline & $\mathrm{Sp}_{4}\left(q_{0}\right)$ & $q=q_{0}^{k}, k$ prime & 2,3 & $b(G)=2$ if $k>2$ \\
\hline & $2^{4} \cdot \mathrm{O}_{4}^{-}(2)$ & $q=p>3$ & 2,3 & $b(G)=3$ iff $G=\mathrm{PGSp}_{4}(5)$ \\
\hline & $S z(q)$ & $p=2, \log _{2} q>1$ odd & 3 & \\
\hline & $\mathrm{L}_{2}(q)$ & $p>3$ & 2 & \\
\hline & $A_{6}$ & $q=p>3$ & 2 & \\
\hline & $A_{7}$ & $q=7$ & 2 & \\
\hline & $\mathrm{O}_{2}^{\epsilon}(q) \prec S_{2}$ & $(\leftrightarrow)$ holds, $\epsilon=-$ if $q=2$ & $2,3,4$ & $b(G)=4$ iff $G=\operatorname{Sp}_{4}(2)^{\prime} \cdot 2^{2}$ \\
\hline & $\mathrm{O}_{2}^{-}\left(q^{2}\right) \cdot 2$ & $(\leftrightarrow)$ holds & 2,3 & \\
\hline \multirow[t]{8}{*}{$\mathrm{L}_{3}(q)$} & $\mathrm{GL}_{1}(q) \prec S_{3}$ & $q>3$ & 2 & see $[\mathbf{1 7}]$ \\
\hline & $\operatorname{GL}_{1}\left(q^{3}\right)$ & & 2,3 & $b(G)=2$ if $q>3$ \\
\hline & $\mathrm{GL}_{3}\left(q_{0}\right)$ & $q=q_{0}^{k}, k$ prime & 2,3 & $b(G)=2$ if $k>2$ \\
\hline & $3^{2} \cdot \mathrm{Sp}_{2}(3)$ & $q=p>5, p \equiv 1(3)$ & 2 & \\
\hline & $\mathrm{O}_{3}(q)$ & $q$ odd & 2,3 & \\
\hline & $\mathrm{U}_{3}\left(q_{0}\right)$ & $q=q_{0}^{2}$ & 2,3 & \\
\hline & $A_{6}$ & $q=4$ & 3 & \\
\hline & $\mathrm{L}_{3}(2)$ & $q=p \equiv 1,2,4(7)$ & 2 & \\
\hline $\mathrm{U}_{3}(q)$ & $\mathrm{GU}_{1}(q) \prec S_{3}$ & & 2,3 & $b(G)=2+\delta_{3, q}+\delta_{4, q}$ \\
\hline \multirow[t]{7}{*}{$(q>2)$} & $\mathrm{GU}_{1}\left(q^{3}\right)$ & & 2 & \\
\hline & $\mathrm{GU}_{3}\left(q_{0}\right)$ & $q=q_{0}^{k}, k>2$ prime & 2 & \\
\hline & $3^{2} \cdot \mathrm{Sp}_{2}(3)$ & $q=p \equiv 2(3)$ & 2 & \\
\hline & $\mathrm{O}_{3}(q)$ & $q$ odd & 2,3 & \\
\hline & $A_{6}$ & $q=5$ & 3 & \\
\hline & $A_{7}$ & $q=5$ & 4 & \\
\hline & $\mathrm{L}_{3}(2)$ & $q=p \equiv 3,5,6(7)$ & $2,3,4$ & $b(G)=2$ if $q>5$ \\
\hline $\mathrm{L}_{2}(q)$ & $\mathrm{GL}_{1}(q) \prec S_{2}$ & $q>5$ & 2,3 & \\
\hline \multirow[t]{4}{*}{$(q>4)$} & $\mathrm{GL}_{1}\left(q^{2}\right)$ & & 2,3 & \\
\hline & $\mathrm{GL}_{2}\left(q_{0}\right)$ & $q=q_{0}^{k}, k>2$ prime & 2 & \\
\hline & $2^{2} \cdot \mathrm{O}_{2}^{-}(2)$ & $q=p>7$ & 2 & \\
\hline & $A_{5}$ & $q \equiv \pm 1(10), q \neq 9$ & 2,3 & $b(G)=2+\delta_{11, q}+\delta_{19, q}$ \\
\hline
\end{tabular}

TABle 6. Base sizes for non-standard actions, II 


\section{References}

1. M. Aschbacher, 'On the maximal subgroups of the finite classical groups', Invent. Math. 76 (1984) 469514.

2. M. Aschbacher and G. M. Seitz, 'Involutions in Chevalley groups over fields of even order', Nagoya Math. J. 63 (1976) 1-91.

3. T. C. Burness, 'Fixed point ratios in actions of finite classical groups, I', J. Algebra, to appear.

4. T. C. Burness, 'Fixed point ratios in actions of finite classical groups, II', J. Algebra, to appear.

5. T. C. Burness, 'Fixed point ratios in actions of finite classical groups, III', J. Algebra, to appear.

6. T. C. Burness, 'Fixed point ratios in actions of finite classical groups, IV', J. Algebra, to appear.

7. T. C. Burness, M. W. Liebeck and A. Shalev, 'Base sizes for simple groups and a conjecture of Cameron', submitted.

8. P. J. Cameron, 'Some open problems on permutation groups', Groups, Combinatorics and Geometry (eds M. W. Liebeck and J. Saxl), LMS Lecture Note Series 165 (Cambridge University Press, 1992), pp. $340-350$.

9. P. J. Cameron and W. M. Kantor, 'Random permutations: some group-theoretic aspects', Combin. Probab. Comput. 2 (1993) 257-262.

10. J. Conway, R. Curtis, S. Norton, R. Parker and R. Wilson, Atlas of Finite Groups (Oxford University Press, 1985).

11. The GAP Group, GAP - Groups, Algorithms, and Programming, Version 4.4, 2004.

12. D. Goldstein and R. M. Guralnick, 'Alternating forms and self-adjoint operators', J. Algebra, to appear.

13. D. Gorenstein, R. Lyons and R. Solomon, The Classification of the Finite Simple Groups, Number 3, Mathematical Surveys and Monographs 40 (Amer. Math. Soc., 1998).

14. R. M. Guralnick and J. Saxl, 'Permutation groups with base size two', in preparation.

15. G. Hiss and G. Malle, 'Low dimensional representations of quasi-simple groups', LMS J. Comput. Math. 4 (2001) 22-63.

16. J. P. JAMES, 'Partition actions of symmetric groups and regular bipartite graphs', Bull. London Math. Soc. 38 (2006) 224-232.

17. J. P. JAmes, 'Two point stabilisers of partition actions of linear groups', J. Algebra 297 (2006) $453-469$.

18. P. B. KLEIDMAN, 'The maximal subgroups of the finite 8-dimensional orthogonal groups $\mathrm{P} \Omega_{8}^{+}(q)$ and of their automorphism groups', J. Algebra 110 (1987) 173-242.

19. P. B. Kleidman, The subgroup structure of some finite simple groups, Ph.D. thesis (University of Cambridge, 1987).

20. P. B. Kleidman and M. W. Liebeck, The Subgroup Structure of the Finite Classical Groups, LMS Lecture Note Series 129 (Cambridge University Press, 1990).

21. R. LAWTher and J. SAXL, 'On the actions of finite groups of Lie type on the cosets of subfield subgroups and their twisted analogues', Bull. London Math. Soc. 21 (1989) 449-455.

22. M. W. Liebeck and A. Shalev, 'Simple groups, permutation groups, and probability', J. Amer. Math. Soc. 12 (1999) 497-520.

23. M. W. Liebeck and A. Shalev, 'Character degrees and random walks in finite groups of Lie type', Proc. London Math. Soc. 90 (2005) 61-86.

24. F. LüBECK, 'Small degree representations of finite Chevalley groups in defining characteristic', LMS J. Comput. Math. 4 (2001) 135-169. 
Timothy C. Burness

St John's College

Oxford OX1 3JP

England

burness@maths.ox.ac.uk
Current address:

Einstein Institute of Mathematics

Hebrew University of Jerusalem

Jerusalem 91904

Israel

burness@math.huji.ac.il 